\title{
Polypeptide Composition and Topology Affect Hydrogelation of Star-Shaped Poly(L-lysine)-Based Amphiphilic Copolypeptides
}

\author{
Thi Ha My Phan ${ }^{1}$, Ching-Chia Huang ${ }^{1}$, Yi-Jen Tsai ${ }^{1}$, Jin-Jia Hu ${ }^{2, *(\mathbb{D}}$ and Jeng-Shiung Jan ${ }^{1,3, *(\mathbb{D})}$ \\ 1 Department of Chemical Engineering, National Cheng Kung University, Tainan 70101, Taiwan; \\ n36087113@gs.ncku.edu.tw (T.H.M.P.); s110076@shsh.tw (C.-C.H.); n36094704@gs.ncku.edu.tw (Y.-J.T.) \\ 2 Department of Mechanical Engineering, National Yang Ming Chiao Tung University, Hsinchu 30010, Taiwan \\ 3 Hierarchical Green-Energy Materials (Hi-GEM) Research Center, National Cheng Kung University, \\ Tainan 70101, Taiwan \\ * Correspondence: jsjan@mail.ncku.edu.tw (J.-J.H.); jjhu@nctu.edu.tw (J.-S.J.)
}

Citation: Phan, T.H.M.; Huang, C.-C.; Tsai, Y.-J.; Hu, J.-J.; Jan, J.-S. Polypeptide Composition and Topology Affect Hydrogelation of Star-Shaped Poly(L-lysine)-Based Amphiphilic Copolypeptides. Gels 2021, 7, 131. https://doi.org/ 10.3390/gels7030131

Academic Editor: Bin Xue

Received: 28 July 2021

Accepted: 27 August 2021

Published: 30 August 2021

Publisher's Note: MDPI stays neutral with regard to jurisdictional claims in published maps and institutional affiliations.

Copyright: (c) 2021 by the authors. Licensee MDPI, Basel, Switzerland. This article is an open access article distributed under the terms and conditions of the Creative Commons Attribution (CC BY) license (https:// creativecommons.org/licenses/by/ $4.0 /)$.

\begin{abstract}
In this research, we studied the effect of polypeptide composition and topology on the hydrogelation of star-shaped block copolypeptides based on hydrophilic, coil poly $(\mathrm{L}-\mathrm{lysine})_{20}(\mathrm{~s}-$ $\mathrm{PLL}_{20}$ ) tethered with a hydrophobic, sheet-like polypeptide segment, which is poly(L-phenylalanine) (PPhe), poly(L-leucine) (PLeu), poly( $\mathrm{L}_{\text {-valine }}$ (PVal) or poly( $\mathrm{L}_{\mathrm{L}}$-alanine) (PAla) with a degree of polymerization (DP) about 5. We found that the PPhe, PLeu, and PVal segments are good hydrogelators to promote hydrogelation. The hydrogelation and hydrogel mechanical properties depend on the arm number and hydrophobic polypeptide segment, which are dictated by the amphiphilic balance between polypeptide blocks and the hydrophobic interactions/hydrogen bonding exerted by the hydrophobic polypeptide segment. The star-shaped topology could facilitate their hydrogelation due to the branching chains serving as multiple interacting depots between hydrophobic polypeptide segments. The 6-armed diblock copolypeptides have better hydrogelation ability than 3-armed ones and $s$-PLL- $b$-PPhe exhibits better hydrogelation ability than s-PLL- $b$-PVal and $s$-PLL- $b$-PLeu due to the additional cation $-\pi$ and $\pi-\pi$ interactions. This study highlights that polypeptide composition and topology could be additional parameters to manipulate polypeptide hydrogelation.
\end{abstract}

Keywords: hydrogels; polypeptide; self-assembly; chain conformation; polymer topology

\section{Introduction}

Hydrogels, which are three-dimensional networks of hydrophilic polymers, are known to contain a large amount of water while maintaining the structures via chemical and physical cross-linking of individual polymer chains. Hydrogels based on natural or synthetic polymers can be yielded via forming non-covalent physical interactions or chemically covalent bonds [1-3]. Due to the ability to mimic animal tissues, their applications in biomedical fields have been rapidly increasing for several decades [4-7]. This has led to a risen request for well-defined hydrogelators with adaptable properties for biomedical applications such as advanced wound healing, drug carriers, tissue engineering scaffolds, and investigating biomechanical functions [8-11]. Moreover, hydrogels have exhibited numerous characteristics such as adjustability, pellucidity, and porosity/permeability. However, most of the known synthetic hydrogels have inferior mechanical properties, compared to some of the natural tissues such as cartilages, tendons, and ligaments [12-14].

According to biocompatibility and chemical versatility, the hydrogels synthesized based on polypeptides are nature-inspired materials that have great potentiality [15-19]. Commonly, the most well-known technique for preparing synthetic polypeptide-based polymers is ring-opening polymerization (ROP), which allows the preparation of homoor copolypeptides with huge volume and superior efficiency [20-23]. A wide range of synthetic polypeptides has shown their capability to adopt secondary conformations such as random coils, $\beta$-sheets, or $\alpha$-helices in an aqueous environment, allowing the adjustment 
of mechanical properties of synthetic polypeptides. Moreover, polypeptides can expand their applications in the biomimetic field by conjugating to other materials such as proteins or nanoparticles [24]. Additionally, synthetic polypeptides possess many functional groups on the side chain, making it possible to modify pre- and post-polymerization [15,23,25].

The self-assembly of amphiphilic copolymers has always been an interesting topic for researchers because of the interconnection of two or more different polymer segments, allowing for the recognition of new material characteristics and the useful application of assembled morphologies. The influence of the balance between hydrophilic and hydrophobic interactions on polypeptide self-assembled morphology has been actively studied. The effect of specific interactions and secondary conformations on block copolypeptides can also be a factor governing polypeptide self-assembly [11,12,16,19,26-28]. Herein, we report the synthesis and hydrogelation of star-shaped diblock copolypeptides based on hydrophilic, coil poly(L-lysine) (s-PLL) blocked with a hydrophobic, sheet-like polypeptide segment, anticipating the hydrogelation. The research progresses to synthesize star-shaped diblock copolypeptides, which have attracted much attention because of their outstanding advantages. A few studies have reported that the star-shaped diblock copolypeptides, which are traditionally synthesized by using dendrimers bearing amino groups as the initiators, could form hydrogels in an aqueous solution and the polypeptide topology could be an additional parameter to manipulate their hydrogelation [29-31]. However, the synthesis of dendrimers required numerous and complicated steps. To limit this drawback, we previously applied ROP to synthesize polypeptides using readily available alcohols containing different numbers of $\mathrm{OH}$ group as the initiators and the 1,1,3,3-tetramethylguanidine (TMG) as the promoter $[28,31,32]$. Our previous report demonstrated that the star-shaped PLL copolypeptides grafted with phenyl or indole group could form hydrogels in an aqueous environment [27]. In this study, we turn to a different aspect and study the hydrogelation of star-shaped diblock copolypeptides. Poly(L-phenylalanine) (PPhe), poly(L-alanine) (PAla), poly(L-leucine) (PLeu), and poly ( $\mathrm{L}_{\text {-valine }}$ (PVal) are chosen to be the second block of the star-shaped diblock copolypeptides. It is anticipated that the self-assembly of star-shaped diblock copolypeptides would be different from that of star-shaped graft ones, which could have a direct impact on their hydrogelation. Furthermore, varying the polypeptide composition including arm number and monomer would affect the hydrogelation and gel mechanical strength and the molecular assembly $[33,34]$. In previous studies, researchers reported that the increase of block copolypeptides arm length and arm number led to decreasing in critical gelation concentration (CGC) and increasing in hydrogelation of polymers [29,31], while it had no obvious trend for graft copolypeptides [28]. Moreover, compared to the linear counterparts, the star-shaped ones could create effective inter/intramolecular interactions between the hydrophobic moieties [35]. It is anticipated that polypeptide composition and topology could be additional parameters to manipulate polypeptide hydrogelation.

\section{Results and Discussion}

\subsection{Synthesis and Characterization of Polypeptides}

Star-shaped diblock copolypeptides based on PZLL tethered with four different polypeptide segments (s-PZLL- $b$-PY) were synthesized by sequential ROP of respective NCAs using 3-armed and 6-armed initiators, which are 1,1,1-tri(hydroxymethyl)propane and dipentaerythritol. The synthesis procedures and polypeptide chemical structures are shown in Scheme 1. The block ratio, the number-average weight $\left(M_{n}\right)$, and molecular weight distribution $\left(M_{w} / M_{n}\right)$, based on gel permeation chromatography-light scattering (GPC-LS) and proton nuclear magnetic resonance $\left({ }^{1} \mathrm{H}\right.$ NMR) (Figure S1), were calculated, as shown in Tables S1 and S2. As the s-PZLL first blocks were prepared, a small portion was drawn from the reaction solution and purified for GPC-LS analysis. Based on ${ }^{1} \mathrm{H}$ NMR analysis, the presence of the chemical shifts for all the protons on the initiators and polypeptides confirmed the successful synthesis of the s-PZLL homopolypeptides and s-PZLL- $b$-PY diblock copolypeptides (Figure 1a,b). The block ratio of initiator to ZLL was determined 
based on the ratio of the integrated areas for the methylene protons $\left.\left(-\mathrm{C}_{(} \mathrm{CH}_{2} \mathrm{O}-\right)_{\mathrm{n}}\right)$ on the initiator and the benzyl protons $\left(-\mathrm{OCH}_{2} \mathrm{C}_{6} \mathrm{H}_{5}\right)$ on s-PZLL (Figure 1a). From ${ }^{1} \mathrm{H}$ NMR analysis, the calculated degrees of polymerization (DPs) reached a good agreement with the feed molar ratios of the initiator and ZLL NCA (Table S2). The DPs, which were calculated from ${ }^{1} \mathrm{H}$ NMR spectra also received a good agreement with the molecular weights of the $s$-PZLL determined based on the GPC analysis (Table S1). Based on the ${ }^{1} \mathrm{H}$ NMR spectra (for example, Figure 1b), the integral ratios of the protons of the benzyl group on the polypeptide chain $\left(-\mathrm{COOCH}_{2} \mathrm{C}_{6} \mathrm{H} 5\right.$ and $\left.-\mathrm{CH}_{2} \mathrm{C}_{6} \mathrm{H}_{5}\right)$, the $\alpha$ protons on $s$-PZLL (or $-\left(\mathrm{CH}-\left(\mathrm{CH}_{2}\right)-\right)-$, and the $\delta$ and $\varepsilon$ protons on the PLeu $\left(-\mathrm{CH}\left(\mathrm{CH}_{3}\right)_{2}\right)$ provided block ratios for both blocks, as summarized in Table S2. To remove the Z-group, s-PZLL-b-PY diblock copolypeptides were reacted with $\mathrm{HBr}$ for $30 \mathrm{~min}$ to obtain $s$-PLL- $b$-PY; confirmed by ${ }^{1} \mathrm{H}$ NMR analysis, the percentages of the residual $Z$ group were well below $5 \%$ (Figure $1 \mathrm{c}$ and Figure S1), consistent with a previous study [30]. It is worth noting that the deprotection step would not result in hydrolysis of ester groups and apparent degradation of star-shaped polypeptides based on our previous studies [28,30,32].

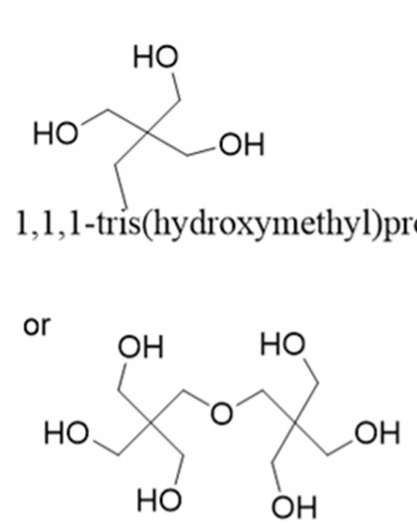

Dipentaerythritol

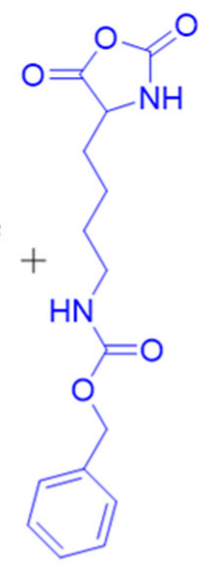

ZLL NCA

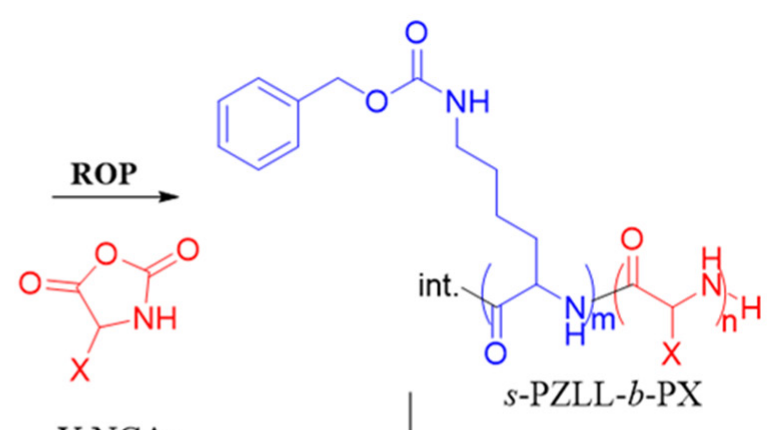

Deprotection of PZLL Dialysis
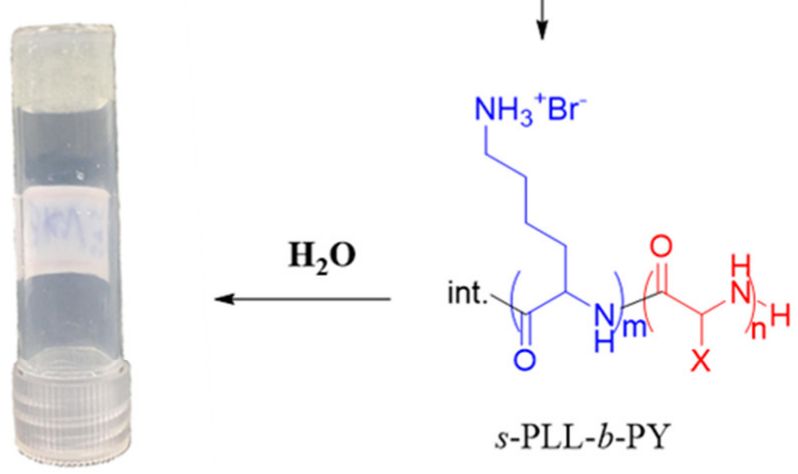

$s$-PLL- $b$-PY

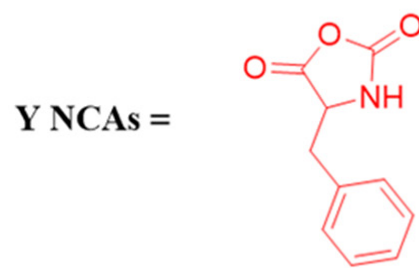

Phe NCA<smiles>CCC(C)CC1NC(=O)OC1=O</smiles>

Leu NCA<smiles>CC1NC(=O)OC1=O</smiles>

Ala NCA<smiles>CC(C)C1NC(=O)OC1=O</smiles>

Val NCA

Scheme 1. The representative scheme for synthesis of star-shaped s-PLL-b-PY diblock copolypeptides and the formation of hydrogels. 


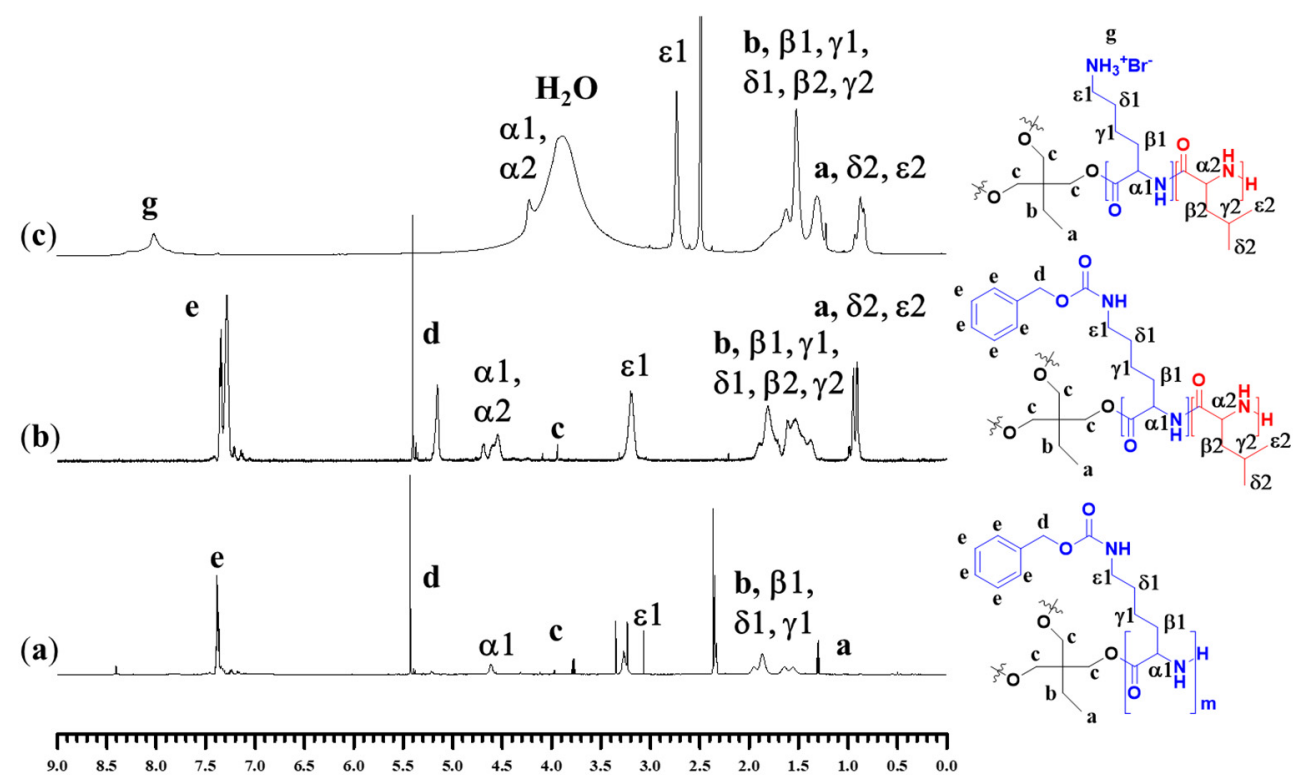

Figure 1. ${ }^{1} \mathrm{H}$ NMR spectra of (a) $3 s-\mathrm{PZLL}_{22}$ in TFA- $d_{1}$, (b) $3 s-\mathrm{PZLL}_{22}-b-\mathrm{PLeu}_{5.5}$ in TFA- $d_{1}$, and (c) $3 s-\mathrm{PLL}_{22}-b-\mathrm{PLeu}_{5.5}$ in DMSO- $d_{6}$.

\subsection{Hydrogelation of Block Polypeptides}

At room temperature (RT), s-PLL- $b$-PY block copolypeptides were dissolved in deionized (DI) water by using ultrasound and vortex mixer. After placing on a counter overnight, the polypeptides can form transparent hydrogels by dispersing in DI water. The CGCs of the polypeptide hydrogels were determined by the tube inverting method, which ranged from 1.0 to $7.0 \mathrm{wt} \%$ (Scheme 1 and Table 1). Rather, it is found that $3 s-\mathrm{PLL}_{22}-b-\mathrm{PAla}_{5.5}$ and 6s-PLL $21-b$-PAla 5.3 diblock copolypeptides formed hydrogels at much higher concentrations, which were 22.0 and $15.0 \mathrm{wt} \%$, respectively. Table 1 showed that the CGC values of the hydrogel samples was in the following order, $6 s-\mathrm{PLL}_{21}-b-\mathrm{PPhe}_{5.3}<6 s-\mathrm{PLL}_{21}-b-\mathrm{PVal}_{5}$ $\sim 6 s-\mathrm{PLL}_{21}-b$-PLeu $4.4<3 s-\mathrm{PLL}_{22}-b$-PPhe $6.3<3 s-\mathrm{PLL}_{22}-b-\mathrm{PVal}_{5.1}<3 s-\mathrm{PLL}_{22}-b$-PLeu 5.5 . It is obvious that the CGCs were dependent on the arm number and composition. It can be seen that the CGCs of 6-armed polypeptide hydrogels were lower than those of 3-armed ones. It can be attributed that the 6-armed polypeptides exhibited more branching chains than the 3-armed ones. The hydrophobic and hydrophilic balance between polypeptide blocks and hydrogen bonding/hydrophobic interactions exerted by the PY segments affected the hydrogelation. $s$-PLL- $b$-PPhe exhibited lower CGC than $s$-PLL- $b$-PVal and $s$-PLL- $b$ PLeu, revealing that the PPhe segment exhibited better hydrogelation ability than the other two segments. It could be attributed that the PPhe segment exhibited additional $\pi-\pi$ interactions between benzyl groups and cation $-\pi$ interactions between PLL and PPhe segments $[30,31,36,37]$.

Table 1. The critical gelation concentration (CGC) and percentages of the different secondary conformations adopted by s-PLL-b-PY diblock copolypeptides by using the fitting software BeStSel.

\begin{tabular}{|c|c|c|c|c|}
\hline \multirow[b]{2}{*}{ Polypeptides } & \multirow[b]{2}{*}{$\begin{array}{c}\text { CGC } \\
(w t \%)\end{array}$} & \multicolumn{3}{|c|}{ Secondary Structure } \\
\hline & & $\begin{array}{c}\text { Random Coil } \\
(\%)\end{array}$ & $\begin{array}{c}\alpha \text {-Helix } \\
(\%)\end{array}$ & $\begin{array}{c}\beta \text {-Sheet } / \beta \text {-Turn } \\
(\%)\end{array}$ \\
\hline $3 s-\mathrm{PLL}_{22}-b-\mathrm{PPhe}_{6.3}$ & 4.5 & 48.3 & 0 & $37.6 / 14.2$ \\
\hline 6s-PLL $21-b-$ PPhe $_{5.3}$ & 1.0 & 48.6 & 0 & $35.8 / 15.6$ \\
\hline $3 s-$ PLL $_{22}-b-$ PLeu $_{5.5}$ & 7.0 & 53.7 & 1.8 & $26.0 / 18.6$ \\
\hline $6 s-\mathrm{PLL}_{21}-b-\mathrm{PLeu}_{4.4}$ & 4.0 & 51.2 & 8.5 & $20.6 / 19.7$ \\
\hline $3 s-\mathrm{PLL}_{22}-b-\mathrm{PVal}_{5.1}$ & 5.5 & 47.2 & 0 & $38.9 / 13.9$ \\
\hline $6 s-\mathrm{PLL}_{21}-b-\mathrm{PVal}_{5}$ & 4.0 & 46.1 & 0 & $39.9 / 14.0$ \\
\hline
\end{tabular}




\subsection{Molecular Structure of Polypeptide Hydrogels}

At neutral conditions, the chain conformations of the star-shaped polypeptides were characterized by circular dichroism (CD) and Fourier transform infrared spectroscopy (FTIR) analyses. The software BeStSel was employed for fitting the CD spectra to compute the secondary conformations adopted by polypeptide chains. The $\mathrm{CD}$ analysis revealed that the star-shaped polypeptides adopted mainly random coil and $\beta$-sheet/turn conformations at neutral conditions (Table 1 and Figure 2a). In agreement with the results determined from the CD analysis, the FTIR spectra of polypeptides exhibited the characteristics of the random coil $\left(1650 \mathrm{~cm}^{-1}\right), \beta$-sheet $\left(1626 \mathrm{~cm}^{-1}\right)$, and $\beta$-turn $\left(1677 \mathrm{~cm}^{-1}\right)$ conformations (Figure 2b). Obviously, the results demonstrated that the chain conformation depended on arm number and the second block of the copolypeptides. It is well known that a random coil conformation would be adopted by the PLL segment at neutral conditions. Evidently, the mole fraction of the second segment was lower than the percentage of $\beta$ sheet/turn conformation. Moreover, 3-armed and 6-armed diblock copolypeptides adopted comparable percentages of both coil and $\beta$-sheet/turn conformations. It demonstrated that PLL segments, due to their confinement, can adopt more ordered conformation by conjugating with a functional moiety on the side chain or a hydrophobic polypeptide on the chain end [38-42]. Rather, the PLL conformation exhibited little dependence on the conjugation of hydrophilic polymer on a PLL segment [16,43]. It is worth noting that a low percentage of $\alpha$-helical conformation adopted by $s$-PLL- $b$-PLeu was detected by CD (Table 1). Previous studies have shown that a hydrophobic PLeu segment with DP higher than 20 would adopt mainly $\alpha$-helical conformation [44,45].

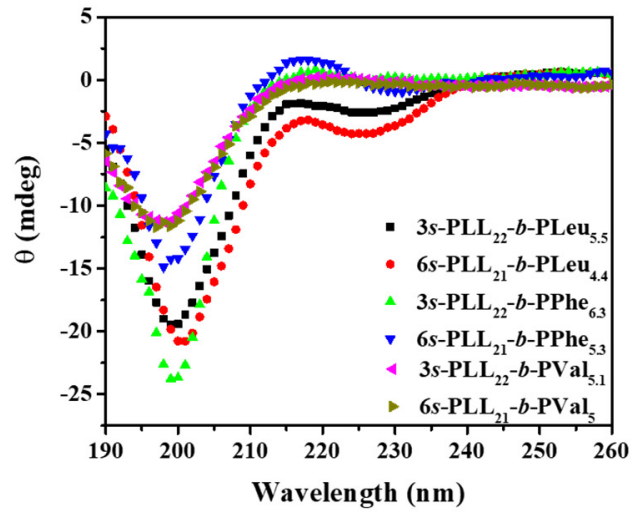

(a)

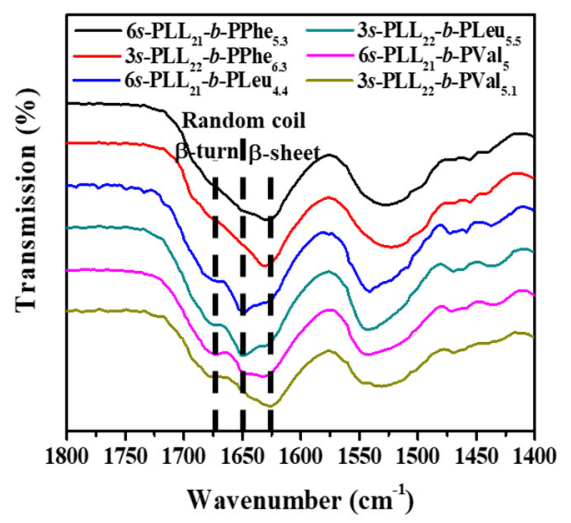

(b)

Figure 2. (a) CD and (b) FTIR spectra of star-shaped s-PLL-b-PY diblock copolypeptides. The polypeptide concentration was $0.1 \mathrm{mg} \mathrm{mL}^{-1}$ for $\mathrm{CD}$ analysis.

\subsection{Morphology and Mechanical Properties of Polypeptide Hydrogels}

To characterize the morphology of hydrogels by scanning electron microscope (SEM), the hydrogel samples were frozen in liquid nitrogen and lyophilized to obtain freezedried samples. The native morphology of samples observed in the free-dried state was demonstrated in the previous studies $[13,14,16,28]$. In Figure 3, all the samples exhibited three-dimensional morphology and porous structure. At the same concentration (5 wt \%), 6s-PLL $21-b$-PPhe 5.3 had a thin elongated fibrillar structure, while 6-armed samples of PLeu and PVal had membranous networks (Figure $3 b, \mathrm{~d}, \mathrm{f})$. Moreover, unlike the 6s-PLL $\mathrm{LL}_{21}-b-$ PPhe $_{6.3}$ sample, $3 s-$ PLL $_{22}-b$-PPhe 6.3 showed membranous morphology. To understand the effect of the arm number and second block on the mechanical strengths of the polypeptide hydrogels, an oscillatory shear rheometer was used to analyze the as-prepared hydrogels formed by $s$-PLL- $b$-PPhe, $s$-PLL- $b$-Pleu, and $s$-PLL- $b$-PVal by conducting angular frequency and strain sweeps (Figure 4). The loss modulus $\mathrm{G}^{\prime \prime}$ was obviously smaller than the storage modulus $G^{\prime}$. Based on the strain sweep test, it showed that the shear-thinning properties were exhibited on all samples, and the gel network was disrupted at higher strain. Some 
samples exhibited the shear-thickening property in the loss modulus $G^{\prime \prime}$, while this phenomenon was not seen in the storage modulus $G^{\prime}$, which was the same case witnessed in the previous studies $[16,28]$. The explanation for the shear-thickening mechanism is still a debatable matter. The increase in loss modulus as the storage modulus decreases at high strain might also reflect the increasing virous and liquid-like character of the hydrogels. It can be possibly attributed to the change of the gel network or the formation of force chains [46]. The mechanical strength of the polypeptide hydrogels with $5.0 \mathrm{wt} \%$ of polypeptide concentration were arranged in this descending order, $6 s-\mathrm{PLL}_{21}-b$-PPhe $5.3>$ $3 s-\mathrm{PLL}_{22}-b-\mathrm{PPhe}_{6.3}>6 s-\mathrm{PLL}_{21}-b-\mathrm{PLeu}_{4.4}>6 s-\mathrm{PLL}_{21}-b-\mathrm{PVal}_{5}$. At a given polypeptide concentration, the 6-armed polypeptide hydrogels exhibited less rigid and higher mechanical strengths than the 3 -armed ones and $6 s-\mathrm{PLL}_{21}-b$-PPhe 5.3 exhibited the highest mechanical strength among all. It can be seen that the mechanical strength of $3 s-\mathrm{PLL}_{22}-b-\mathrm{PPhe}_{6.3}$ hydrogel was higher than those of $6 s-\mathrm{PLL}_{21}-b-\mathrm{PLeu}_{4.4}$ and $6 s-\mathrm{PLL}_{21}-b-\mathrm{PVal}_{5}$ ones. The $3 s-\mathrm{PLL}_{22}-b$-PLeu 5.5 hydrogel exhibited comparable mechanical strength with the 3s-PLL $22-$ $b$-PVal 5.1 one. The results revealed that the hydrogel rigidity and mechanical strength would depend on the polypeptide composition and topology. Upon large-amplitude strain oscillations, the mechanical strength recovery of these hydrogels was monitored for the investigation of the gel recovery. All the samples demonstrated their ability to progressively recover more than $98 \%$ of their original strength after the removal of shearing (Figure 5). The data showed that these star-shaped polypeptide hydrogels have the capability to recover from sol to gel.

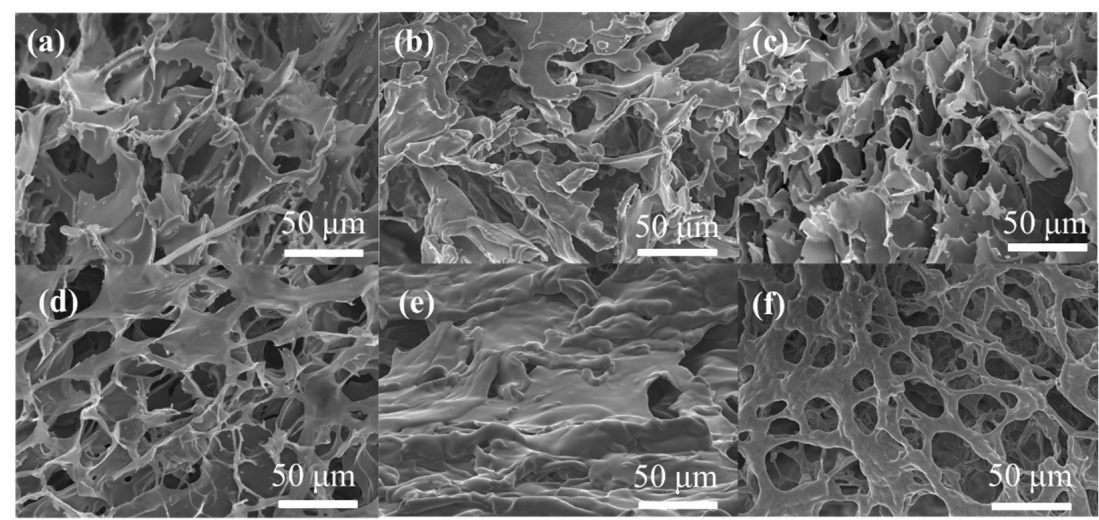

Figure 3. SEM images of freeze-dried (a) 3s-PLL $22-b-\mathrm{PLeu}_{5.5}(8.0 \mathrm{wt} \%)$, (b) $6 s-\mathrm{PLL}_{21}-b-\mathrm{PLeu}_{4.4}$ $(5.0 \mathrm{wt} \%)$, (c) $3 s-\mathrm{PLL}_{22}-b-\mathrm{PVal}_{5.1}(8.0 \mathrm{wt} \%)$, (d) $6 s-\mathrm{PLL}_{21}-b-\mathrm{PVal}_{5}(5.0 \mathrm{wt} \%)$, (e) $3 s-\mathrm{PLL}_{22}-b-\mathrm{PPhe}_{6.3}$ $(5.0 \mathrm{wt} \%)$ and (f) $6 \mathrm{~s}-\mathrm{PLL}_{21}-b-\mathrm{PPhe}_{5.3}(5.0 \mathrm{wt} \%)$ hydrogel samples.

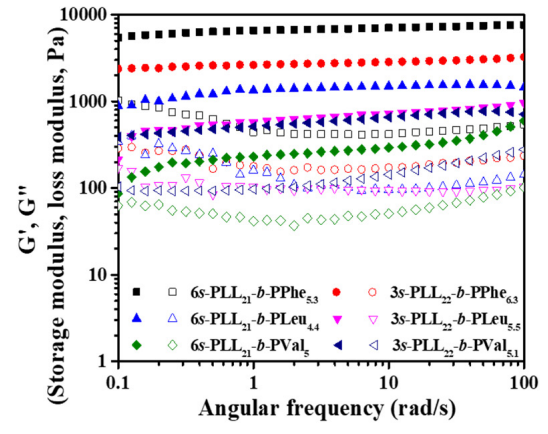

(a)

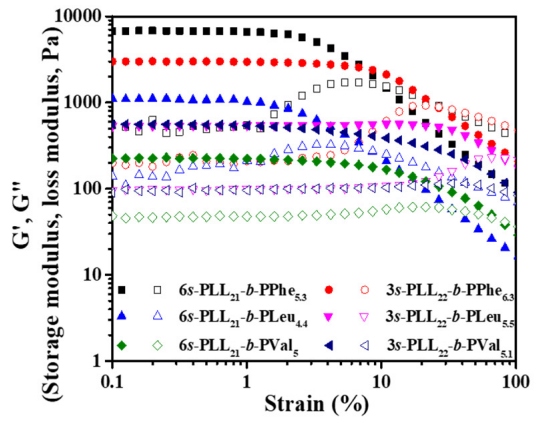

(b)

Figure 4. (a) Storage modulus $\mathrm{G}^{\prime}$ (solid symbols) and loss modulus $\mathrm{G}^{\prime \prime}$ (open symbols) of 6s-PLL 21 - $b$ PPhe $_{5.3}(5.0 \mathrm{wt} \%), 3 s-\mathrm{PLL}_{22}-b-\mathrm{PPhe}_{6.3}(5.0 \mathrm{wt} \%), 6 s-\mathrm{PLL}_{21}-b-\mathrm{PLeu}_{4.4}(5.0 \mathrm{wt} \%), 3 s-\mathrm{PLL}_{22}-b-\mathrm{PLeu}_{5.5}$ $(8.0 \mathrm{wt} \%), 6 s-\mathrm{PLL}_{21}-b-\mathrm{PVal}_{5}(5.0 \mathrm{wt} \%)$, and $3 s-\mathrm{PLL}_{22}-b-\mathrm{PVal}_{5.1}(8.0 \mathrm{wt} \%)$ hydrogels as a function of angular frequency at strain $=0.1 \%$; (b) strain sweeps for the same samples at frequency $=1.0 \mathrm{rad} / \mathrm{s}$. 


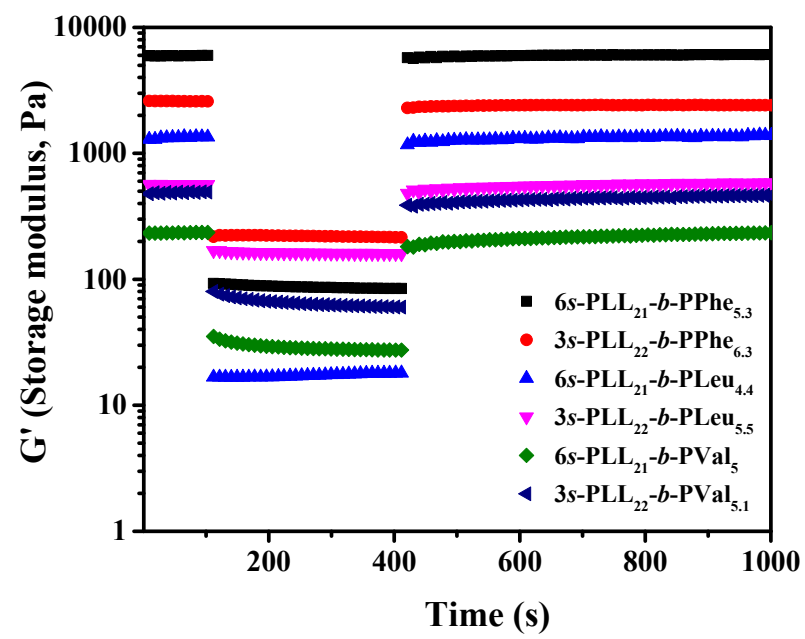

Figure 5. Storage modulus $\mathrm{G}^{\prime}$ as a function of time for $6 s-\mathrm{PLL}_{21}-b-\mathrm{PPhe}_{5.3}(5.0 \mathrm{wt} \%), 3 s-\mathrm{PLL}_{22}-b-$ PPhe $_{6.3}(5.0 \mathrm{wt} \%), 6 s-\mathrm{PLL}_{21}-b-\mathrm{PLeu}_{4.4}$ (5.0 wt\%), 3s-PLL 22-b-PLeu 5.5 (8.0 wt\%), 6s-PLL $21-b-\mathrm{PVal}_{5}$ $(5.0 \mathrm{wt} \%)$, and $3 s-\mathrm{PLL}_{22}-b-\mathrm{PVal}_{5.1}(8.0 \mathrm{wt} \%)$ hydrogel samples. At $100 \mathrm{~s}$, the gel structure was broken down at a constant strain of $100 \%$ and an angular frequency of $1.0 \mathrm{rad} / \mathrm{s}$ by applying nonlinear large-amplitude oscillations. Then, the mechanical strength recovery of the hydrogel samples was monitored at a constant strain of $1 \%$ and an angular frequency of $1.0 \mathrm{rad} / \mathrm{s}$.

\subsection{Molecular Assembly of Polypeptide Hydrogels}

$\mathrm{X}$-ray diffraction analysis $(\mathrm{XRD})$ analysis was employed to determine the macromolecular structure of the freeze-dried hydrogel samples. The presence of a broad peak at $2 \theta>20^{\circ}$ in the freeze-dried samples demonstrated the presence of an amorphous phase in the samples (Figure S2). Small-angle X-ray scattering (SAXS) analysis was employed to elucidate the packing morphology of the sol and gel samples in the nanometer scale. From SAXS profiles, the region $0.02 \AA^{-1}<\mathrm{q}<0.07 \AA^{-1}$ was selected to decide the slope and shape factor. Upon the sol-to-gel transition, all the samples except 3s-PLL ${ }_{22}-b-\mathrm{PLeu}_{5.5}$ exhibited the slope (n) transformed from -2 to almost -3.5 in the scattering intensity $I(q) \propto q^{\mathrm{n}}$, while the 3s-PLL $22-b$-PLeu 5.5 sample exhibited the slope (n) transformed from -1 to almost -3.5 (Figure 6 and Figure S3). The results suggested all the hydrogel samples self-assembled to form ill-defined three-dimensional (3D) nano-assemblies. In the sol state, all the samples except 3s-PLL $22-b$-PLeu 5.5 formed two-dimensional (2D) nano-assemblies $[47,48]$. Rather, $3 s-P_{22}-b$-PLeu 5.5 self-assembled to form one-dimensional (1D) fibrillar morphology, analogous to the study by Pine et al., showing that of the linear PLL- $b$-PLeu polypeptide hydrogels comprised of 1D twisted fibrils [33]. In order to quantify the nano-assemblies, SASview software was used to fit the SAXS profiles of 6s-PLL- $b$-PY hydrogel samples in the region $0.02 \AA^{-1}<\mathrm{q}<0.2 \AA^{-1}$ (Figure S3). The preliminary results showed that the radii of gyration $\left(\mathrm{R}_{\mathrm{g}}\right)$ of the nano-assemblies in the $6 s-\mathrm{PLL}_{21}-b$-PPhe ${ }_{5.3}, 6 s-\mathrm{PLL}_{21}-b$-PLeu $\mathrm{PL}_{4.4}$, and $6 s-\mathrm{PLL}_{21}-b-\mathrm{PVal}_{5}$ hydrogels were calculated to be $58.5,97.5$, and $47.6 \AA$, respectively (Table 2). The SAXS profiles of 3s-PLL-b-PY hydrogel samples cannot be fitted by SASview software due to the presence of a peak in the region $0.02 \AA^{-1}<\mathrm{q}<0.03 \AA^{-1}$, which can be used to calculate the characteristic $d$ spacing. The $d$ spacing values for $3 s-P L L_{22}-b-$ $\mathrm{PVal}_{5.1}, 3 s-\mathrm{PLL}_{22}-b-\mathrm{PPhe}_{6.3}$, and 3s-PLL $22-b-\mathrm{PLeu}_{5.5}$ hydrogels were 314,273 , and $241.5 \AA$, respectively (Table S3 and Figure S3), which could possibly be the distance between the nano-assemblies or other ordered spacing. 

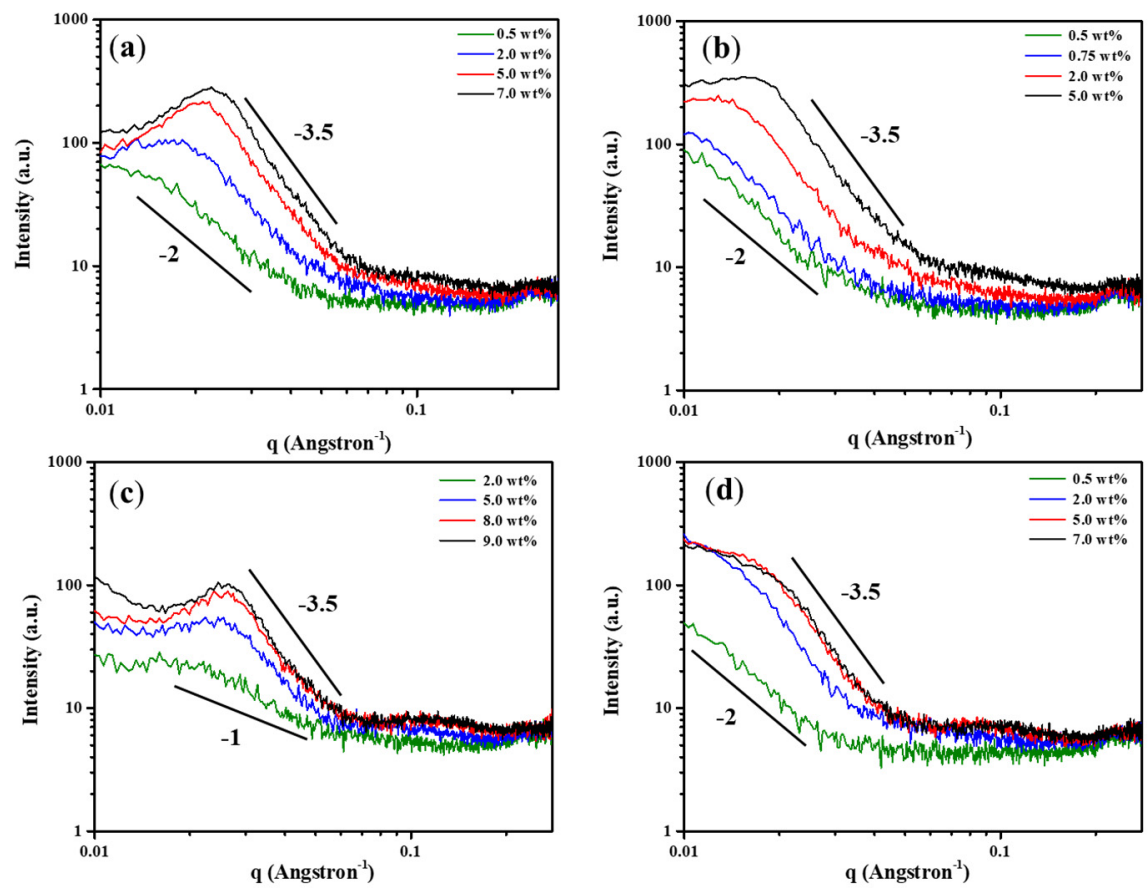

Figure 6. SAXS profiles of (a) $3 s-\mathrm{PLL}_{22}-b$-PPhe $_{6.3}$, (b) $6 s-\mathrm{PLL}_{21}-b$-PPhe 5.3 , (c) $3 s-\mathrm{PLL}_{22}-b$-PLeu 5.5 , and (d) $6 s-\mathrm{PLL}_{21}-b$-PLeu $4.4 \mathrm{sol}$ and gel solutions at different polypeptide concentrations in DI water.

Table 2. The radii of gyration $\left(\mathrm{R}_{\mathrm{g}}\right)$ obtained from fitting SAXS profiles of 6s-PLL- $b$-PY hydrogel samples by using SASview software.

\begin{tabular}{ccc}
\hline Samples & Concentration $(\mathbf{w t} \%)$ & $\operatorname{Rg}(\AA)$ \\
\hline 6s-PLL $20-b-$ PPhe $_{5}$ & 5.0 & 58.5 \\
6s-PLL $20-b-\mathrm{PLeu}_{4.2}$ & 5.0 & 97.5 \\
6s-PLL $20-\mathrm{PVal}_{4.8}$ & 5.0 & 47.6 \\
\hline
\end{tabular}

\subsection{Gelation Mechanism of Polypeptide Hydrogels}

The above results implied that the hydrophobic, sheet-like PPhe, PLeu, and PVal segments are good hydrogelators to promote hydrogelation of star-shaped PLL-b-PY diblock copolypeptides. The hydrophobic and hydrophilic balance between two segments and the hydrophobic interactions/hydrogen bonding adopted by the second blocks affected the formation of hydrogels. The polypeptide topology and composition were the two main factors to affect the non-covalent interactions, which are hydrogen bonding and hydrophobic interactions, and the amphiphilic nature exhibited by these diblock copolypeptides. We found that linear PLL- $b$-PPhe diblock copolypeptides with DP lower than 30 could not form hydrogels in an aqueous solution at the polypeptide concentration below $10 \mathrm{wt} \%$. This study highlighted that star-shaped topology could facilitate the hydrogelation of $s$-PLL- $b$ PY diblock copolypeptides due to the multiple interacting depots between PY segments. It can be seen that the 6-armed diblock copolypeptides had greater hydrogelation ability, as compared to 3-armed ones, which is due to the fact that each 6-armed polypeptide chain has more inter-chain interacting depots than the 3-armed one. s-PLL-b-PPhe exhibited lower CGC than $s$-PLL- $b$-PVal and $s$-PLL- $b$-PLeu due to the additional $\pi-\pi$ interactions between benzyl groups on the PPhe segment and cation- $\pi$ interactions between PLL and PPhe segments. Moreover, it is obvious that all the hydrogel samples could form 3D nano-assemblies by self-assembly and adopt different conformations from each segment. The difference of morphology between s-PLL-b-PPhe and the others, between 3-armed and 6-armed samples of PPhe, demonstrated the effects of hydrophobic component composition and arm number on the structure of polymers. Our previous study showed that CGCs of $3 s-\mathrm{PLL}_{20}-\mathrm{g}-\mathrm{PPhe}_{0.3}$ and $6 s-\mathrm{PLL}_{20}-\mathrm{g}-\mathrm{PPhe}_{0.3}$ graft copolypeptides were 5.0 and $7.0 \mathrm{wt} \%$, 
respectively $[28,32]$, which were higher than those of the corresponding s-PLL-b-PPhe with the same arm number in this study. Obviously, the hydrogelation of $s$-PLL-b-PPhe diblock copolypeptides is more effective than the graft copolopeptides, which could be attributed to the efficient packing of $s$-PLL- $b$-PPhe. Consequently, s-PLL- $b$-PPhe could self-assemble to form interconnected, membraneous networks to accommodate water molecules at relatively lower polypeptide concentrations.

\section{Conclusions}

We demonstrate that star-shaped s-PLL- $b$-PPhe, $s$-PLL- $b$-PVal, and $s$-PLL- $b$-PLeu diblock copolypeptides but not PLL-b-PAla can self-assemble to form hydrogels with CGCs ranged between 1.0 to $7.0 \mathrm{wt} \%$, depending on the arm number and hydrophobic, sheetlike polypeptide segment. The amphiphilic balance between polypeptide blocks and the hydrogen bonding/hydrophobic interactions exerted by the hydrophobic polypeptide segment dictated the hydrogelation and mechanical properties of the hydrogels. Based on the different non-covalent interactions (hydrophobic, hydrophilic and, hydrogen bonding), the formation of multiple interacting depots could facilitate hydrogelation at low polypeptide concentrations. The experimental data showed that the CGCs of 6-armed diblock copolypeptides were lower than those of 3-armed ones and 6s-PLL $21-b$-PPhe 5.3 exhibited the lowest CGC value (1.0 $\mathrm{wt} \%$ ) among all. The additional $\pi-\pi$ and cation $-\pi$ interactions rendered the $s$-PLL- $b$-PPhe exhibiting better hydrogelation ability than $s$-PLL$b$-PVal and s-PLL- $b$-PLeu. This study clearly illustrated that the combination of a sheet-like segment as a hydrogelator and star-shaped topology can trigger the hydrogelation of these block copolypeptides.

\section{Materials and Methods}

\subsection{Materials}

Schlenk-line techniques were used to handle all compounds, which are sensitive to air and moisture under a nitrogen atmosphere. All chemicals and solvents were ACS reagent grade and used without any purification process unless otherwise noted. $\mathrm{N}, \mathrm{N}$ dimethylformamide (DMF, Macron Fine Chemicals ${ }^{\mathrm{TM}}$, Radnor, PA, USA), hexane (ECHO CHEMICAL CO., LTD, Miaoli County, Taiwan), and THF (J.T.Baker, Phillipsburg, NJ, USA) were dried by 4A molecular sieves (UniRegion Bio-Tech Co., Hsinchu, Taiwan), calcium hydride (90-95\%, Alfa Aesar, Shanghai, China), and sodium metal $(99.95 \%$, in mineral oil, Sigma-Aldrich, St. Quentin Fallavier, France), respectively. Z-L-lysine (ZLL) (Sigma-Aldrich, Schaffhausen, Switzerland), L-leucine (Leu) (Sigma-Aldrich, Tokyo, Japan), L-phenylalanine (Phe) (Sigma-Aldrich, Tokyo, Japan), L-alanine (Ala) (Sigma-Aldrich, Taufkirchen, Germany), and L-valine (Val) (Sigma-Aldrich, St. Louis, MO, USA) Ncarboxyanhydrides (NCAs) were prepared based on a procedure reported in the previous papers [28,32].

\subsection{Synthesis of Star-Shaped Poly(L-lysine)-Based Block Copolypeptides}

Following the reported procedure, an initiator was used for the synthesis process of the diblock copolypeptides to sequentially polymerize ZLL and other NCAs $[28,32,49]$. The block ratio was set to be 4:1. The feed molar ratio of 3-armed initiator (1,1,1-tris(hydroxymethyl) propane) (Acros Organics, Schwerte, Germany) to ZLL NCA was set to be 1:60. The feed molar ratio of 6-armed initiator (dipentaerythritol) (Acros Organics, Shanghai, China) to ZLL NCA was set to be 1:120. A detailed procedure for the synthesis of 3-armed-poly(Z-LLysine) ${ }_{20}$-block-poly(L-phenylalanine) ${ }_{5}\left(3 s-\mathrm{PZLL}_{20}-b\right.$-PPhe $\left.{ }_{5}\right)$ is given below as an example.

In a glove box, ZLL $(2.0 \mathrm{~g})$ and 3-armed initiator $(14.6 \mathrm{mg})$ were dissolved in anhydrous DMF (6.54 mL and $5.45 \mathrm{~mL}$, respectively) in round bottom flasks. A TMG stock solution (6.0 mM, $24.62 \mu \mathrm{L}$, Aldrich, China) was dropped into the initiator solution. The solution was heated and stirred until becoming transparent. Then, the ZLL NCA solution was added to the completely dissolved initiator solution. The mixture was stirred outside the glove box at RT under a nitrogen atmosphere for $48 \mathrm{~h}$. Phe NCA $(312.4 \mathrm{mg})$ was dissolved 
in anhydrous DMF (1.64 mL) and poured into the reaction mixture. Upon stirring for an additional $24 \mathrm{~h}$, the reaction mixture was dialyzed against DMF, methanol (ECHO, Taiwan), and DI water for $2 \mathrm{~h}, 24 \mathrm{~h}$, and $72 \mathrm{~h}$, respectively, using cellulose membrane tubes (MWCO $6000-8000 \mathrm{~g} \mathrm{~mol}^{-1}$, Spectrum Laboratories, USA). A white product was collected after the lyophilization of the dialyzed solution (yield: $80-90 \%$ ).

The deprotecting reaction was conducted by using hydrobromic acid ( $\mathrm{HBr}$ ) (Acros, Israel) to remove the $\mathrm{Z}$ group. As a reference procedure, the polypeptide (1.5 g) was completely dissolved in trifluoroacetic acid (TFA, $75 \mathrm{~mL}$, Alfa Aesar, Lancashire, UK) in a round bottom flask. A 5-fold excess with respect to the $\mathrm{Z}$ group molar ratio of a $33 \mathrm{wt} \% \mathrm{HBr}$ solution in acetic acid was added to the solution slowly $[16,28,31,32]$. The reaction mixture was precipitated into diethyl ether (ECHO, Taiwan) after stirring for $30 \mathrm{~min}$ at RT. The precipitate was collected by using centrifugation and washed twice with diethyl ether. After drying under a vacuum, DI water was used to dissolve the collected product. Then, the solution was poured into dialysis membranes (MWCO 6000-8000 $\mathrm{g} \mathrm{mol}^{-1}$ ) and dialyzed against DI water for $72 \mathrm{~h}$. A white spongy product was collected after freeze-drying (yield: 90-95\%).

\subsection{Characterization of Block Polypeptides}

The polypeptides were characterized by proton nuclear magnetic resonance $\left({ }^{1} \mathrm{H}-\mathrm{NMR}\right)$ and gel permeation chromatography-light scattering (GPC-LS). s-PZLL dissolved in TFA- $d_{1}$ and $s-$ PLL- $b$-PY dissolved in DMSO- $d_{6}$ were analyzed by using a BRUKER ADVANCE III HD NMR (600 MHz, Bruker Corporation, Karlsruhe, Germany). The 3-armed and 6-armed PZLL homopolypeptides were analyzed by a Viscotek GPS-LS system (Malvern Instruments Limited, Worcestershire, UK) equipped with two ViscoGEL I-Series columns (catalog number: I-MBHMW-3078 and I-MBLMW-3078, Viscotek) and three detectors, which are Dual 270 viscometer, right-angle light scattering, and VE3580 RI for efficient separation. That GPS-LS system was performed at $55^{\circ} \mathrm{C}$ and $1.0 \mathrm{~mL} \mathrm{~min}{ }^{-1}$ of flow rate on the star-shaped PZLL homopolypeptides. The eluent was DMF containing $0.1 \mathrm{M} \mathrm{LiBr}$ (Alfa Aesar, Shanghai, China), and the calculation standard was polystyrene (molecular weight: 25,000 $\mathrm{g} \mathrm{mol}^{-1}$, Alfa Aesar, Shanghai, China). The 3-armed and 6-armed PZLL homopolypeptides were dissolved in DMF completely. Before GPC-LS analysis, those solutions were passed through a $0.2 \mu \mathrm{m}$ PTFE filter (13 mm, Finetech, Taiwan). The equipped software (OmniSEC 4., Viscotek, Malvern Instruments Limited, Worcestershire, UK) was employed to determine their number-average molecular weights $\left(M_{n}\right)$ and molecular weight distributions $\left(M_{w} / M_{n}\right)$.

\subsection{Preparation Polypeptide Hydrogels and Determination of Critical Gelation Concentration (CGC)}

At RT, the freeze-dried diblock copolypeptide samples were completely dissolved in DI water by using the ultrasound and vortex mixer to obtain clear solutions. Then, the resultant mixtures were placed on a counter overnight at RT to reach equilibrium. The vial inverting method was employed for determining the CGC of the samples $[26,50,51]$. Following the above procedure, the hydrogels were prepared with provided concentrations in $5 \mathrm{~mL}$ vials. The CGCs were determined as the samples did not flow for $60 \mathrm{~s}$ after the vials were inverted.

\subsection{Characterization of Polypeptide Secondary Conformation}

Circular dichroism (CD), X-ray diffraction (XRD), small-angle X-ray scattering (SAXS), and Fourier transform infrared (FTIR) were employed to measure the polypeptide secondary conformation and self-assembled structures. CD measurements of polypeptide samples were conducted at the concentration of $0.1 \mathrm{mg} \mathrm{mL}^{-1}$ on a JASCO J-815 spectrometer (JASCO Corporation, Japan) in a $0.1 \mathrm{~mm}$ quartz cell from $190 \mathrm{~nm}$ to $260 \mathrm{~nm}$ of the wavelength. A Rigaku Ultima IV-9407F701 X-ray spectrometer (Rigaku Corporation, Japan) was used to record XRD patterns of star-shaped polypeptide hydrogel samples. XRD system scanned the patterns from $2 \theta=5^{\circ}$ to $40^{\circ}$ at a speed of $10^{\circ} \mathrm{min}^{-1}$ by using radiation 
(50 kV, $250 \mathrm{~mA}$ ) and $\mathrm{Cu} \mathrm{K}$ alpha $(0.154 \mathrm{~nm})$. At $25^{\circ} \mathrm{C}$, SAXS profiles of the sol and gel samples were obtained under $4 \times 10^{-1}$ torr and the voltage and current were controlled in $45 \mathrm{kV}$ and $650 \mu \mathrm{A}$, respectively. The SAXS measurements were run on a Bruker diffractometer (NanoSTAR-SHAPED U system, Bruker AXS GmbH, Germany). The samples were contained in $1 \mathrm{~mm}$ quartz capillary tubes at different sol and gel concentrations. Before the measurements, a silver behenate was used as a standard sample for calibrating the SAXS. Attenuated total reflectance FTIR spectra of freeze-dried gel samples were recorded on a Thermo Nicolet Nexus 670 FTIR spectrometer (Thermo Electron Corporation, USA).

\subsection{Characterization of Polypeptide Hydrogels}

A Hitachi SU8010 microscope (Hitachi High-Technologies Corporation, Japan) was used to take the field emission scanning electron microscopy (FE-SEM) images of freezedried samples. For rheological measurements, the s-PLL- $b$-PY polypeptide hydrogel samples were prepared at $5.0 \mathrm{wt} \%$ and $8.0 \mathrm{wt} \%$ of polypeptide concentration and tested at RT with a $25 \mathrm{~mm}$ diameter aluminum plate geometry. By varying angular frequency and strain amplitude, a DISCOVERY HR-2 (TA Instruments/Waters Corporation, USA) controlled strain rheometer was used to measure the dynamic variety on loss modulus $\left(G^{\prime \prime}\right)$ and storage modulus $\left(\mathrm{G}^{\prime}\right)$ of the polypeptide hydrogel samples. To perform the hydrogel recovery measurements, the polypeptide hydrogel structures were broken down by these steps: nonlinear, large-amplitude oscillations ( $100 \%$ of strain) at an angular frequency of $1.0 \mathrm{rad} \mathrm{s}^{-1}$ for $300 \mathrm{~s}$, followed by monitoring the mechanical strength recovery of hydrogels at the same angular frequency with a constantly low strain of $1 \%$.

Supplementary Materials: The following are available online at https://www.mdpi.com/article/ 10.3390/gels7030131/s1, Table S1: Feed molar ratio, degree of polymerization (DP), number average molecular weight $\left(\mathrm{M}_{n}\right)$, and molecular weight distribution $\left(M_{w} / M_{n}\right)$ of $s$-PZLL homopolypeptides, Table S2: Feed ratios and block ratios between the ZLL block and the second block, Table S3: The corresponding d spacing obtained from SAXS profiles of 3-armed PLL-b-PY hydrogel samples, Figure S1: ${ }^{1} \mathrm{H}-\mathrm{NMR}$ spectra of (a) $6 s-\mathrm{PLL}_{21}-b-\mathrm{PVal}_{5}$ in DMSO-d ${ }_{6}$, (b) $6 s-\mathrm{PLL}_{21}-b-\mathrm{PLeu}_{4.4}$ in DMSO- $\mathrm{d}_{6}$, and (c) 6s-PLL ${ }_{21}-b$-PPhe 5.3 in DMSO- $\mathrm{d}_{6}$, Figure S2: XRD patterns of freeze-dried 6s-PLL 21 -b-PPhe 5.3 (5.0 wt\%), 3s-PLL 22 -b-PPhe 6.3 (5.0 wt\%), 6s-PLL 21 -b-PLeu 4.4 (5.0 wt $\%$ ), 3s-PLL $22-$ b-PLeu $5.5(8.0 \mathrm{wt} \%)$, 6s-PLL 21 -b-PVal 5 (5.0 wt\%), and 3s-PLL $22-\mathrm{b}^{-\mathrm{PVal}} 5.1$ (8.0 wt \%) hydrogel samples, Figure S3: SAXS profiles of (a) $3 s-\mathrm{PLL}_{22}-b-\mathrm{PVal}_{5.1}$ and (b) $6 s-\mathrm{PLL}_{21}-b-\mathrm{PVal}_{5}$ sol and gel solutions at different polypeptide concentrations in DI water.

Author Contributions: Conceptualization, J.-J.H. and J.-S.J.; methodology, T.H.M.P.; validation, C.-C.H. and Y.-J.T.; formal analysis, T.H.M.P., C.-C.H. and Y.-J.T.; investigation, T.H.M.P. and C.-C.H.; resources, J.-S.J.; data curation, T.H.M.P.; writing—original draft preparation, T.H.M.P.; writingreview and editing, J.-J.H.; visualization, J.-J.H. and C.-C.H.; supervision, J.-S.J.; project administration, J.-S.J.; funding acquisition, J.-S.J. All authors have read and agreed to the published version of the manuscript.

Funding: This research was funded by the Ministry of Science and Technology, Taiwan (MOST 107-2923-M-006-002-MY3 and 108-2221-E-006-034-MY3). This work was financially supported by the Hierarchical Green-Energy Materials (Hi-GEM) Research Center, from The Featured Areas Research Center Program within the framework of the Higher Education Sprout Project by the Ministry of Education (MOE) and the Ministry of Science and Technology (MOST 110-2634-F-006-017) in Taiwan. We thank Ms. Bi-Yun Lin and Mr. Kun-Hsu Lee (Instrument Center, National Cheng Kung University) for their help in performing the NMR and SAXS experiments, respectively.

Institutional Review Board Statement: Not applicable.

Informed Consent Statement: Not applicable.

Data Availability Statement: The data presented in this study are available on request from the corresponding author. 
Acknowledgments: The authors acknowledge the financial support from the Ministry of Science and Technology, Taiwan, and Bi-Yun Lin of the machine equipment belonging to the Instrument Center of National Cheng Kung University.

Conflicts of Interest: The authors declare no conflict of interest.

\section{References}

1. Appel, E.A.; del Barrio, J.; Loh, X.J.; Scherman, O.A. Supramolecular polymeric hydrogels. Chem. Soc. Rev. 2012, 41, 6195-6214. [CrossRef]

2. Anderson, A.J.; Culver, H.R.; Bryant, S.J.; Bowman, C.N. Viscoelastic and thermoreversible networks crosslinked by non-covalent interactions between "clickable" nucleic acid oligomers and DNA. Polym. Chem. 2020, 11, 2959-2968. [CrossRef]

3. Strandman, S.; Zhu, X. Self-healing supramolecular hydrogels based on reversible physical interactions. Gels $2016,2,16$. [CrossRef]

4. Barrett-Catton, E.; Ross, M.L.; Asuri, P. Multifunctional Hydrogel Nanocomposites for Biomedical Applications. Polymers 2021, 13, 856. [CrossRef] [PubMed]

5. Pham, T.-N.; Su, C.-F.; Huang, C.-C.; Jan, J.-S. Biomimetic hydrogels based on L-Dopa conjugated gelatin as pH-responsive drug carriers and antimicrobial agents. Colloids Surf. B Biointerfaces 2020, 196, 111316. [CrossRef] [PubMed]

6. Hogan, K.J.; Mikos, A.G. Biodegradable thermoresponsive polymers: Applications in drug delivery and tissue engineering. Polymer 2020, 211, 123063. [CrossRef]

7. Gao, Q.; Zhang, C.; Wang, M.; Wu, Y.; Gao, C.; Zhu, P. Injectable pH-responsive poly ( $\gamma$-glutamic acid)-silica hybrid hydrogels with high mechanical strength, conductivity and cytocompatibility for biomedical applications. Polymer 2020, 197, 122489. [CrossRef]

8. Ravichandran, R.; Sundarrajan, S.; Venugopal, J.R.; Mukherjee, S.; Ramakrishna, S. Advances in polymeric systems for tissue engineering and biomedical applications. Macromol. Biosci. 2012, 12, 286-311. [CrossRef]

9. Li, Y.; Rodrigues, J.; Tomás, H. Injectable and biodegradable hydrogels: Gelation, biodegradation and biomedical applications. Chem. Soc. Rev. 2012, 41, 2193-2221. [CrossRef]

10. Pham, T.-N.; Jiang, Y.-S.; Su, C.-F.; Jan, J.-S. In situ formation of silver nanoparticles-contained gelatin-PEG-dopamine hydrogels via enzymatic cross-linking reaction for improved antibacterial activities. Int. J. Biol. Macromol. 2020, 146, 1050-1059. [CrossRef] [PubMed]

11. Chen, Y.-F.; Chen, G.-Y.; Chang, C.-H.; Su, Y.-C.; Chen, Y.-C.; Jiang, Y.-s.; Jan, J.-S. TRAIL encapsulated to polypeptide-crosslinked nanogel exhibits increased anti-inflammatory activities in Klebsiella pneumoniae-induced sepsis treatment. Mater. Sci. Eng. C. 2019, 102, 85-95. [CrossRef]

12. Hsu, F.-M.; Hu, M.-H.; Jiang, Y.-S.; Lin, B.-Y.; Hu, J.-J.; Jan, J.-S. Antibacterial polypeptide/heparin composite hydrogels carrying growth factor for wound healing. Mater. Sci. Eng. C 2020, 112, 110923. [CrossRef]

13. Sun, Y.; Wollenberg, A.L.; O'Shea, T.M.; Cui, Y.; Zhou, Z.H.; Sofroniew, M.V.; Deming, T.J. Conformation-directed formation of self-healing diblock copolypeptide hydrogels via polyion complexation. J. Am. Chem. Soc. 2017, 139, 15114-15121. [CrossRef]

14. Huang, J.; Hastings, C.L.; Duffy, G.P.; Kelly, H.M.; Raeburn, J.; Adams, D.J.; Heise, A. Supramolecular hydrogels with reverse thermal gelation properties from (oligo) tyrosine containing block copolymers. Biomacromolecules 2013, 14, 200-206. [CrossRef]

15. O’Brien, S.; Brannigan, R.P.; Ibanez, R.; Wu, B.; O’Dwyer, J.; O’Brien, F.J.; Cryan, S.-A.; Heise, A. Biocompatible polypeptide-based interpenetrating network (IPN) hydrogels with enhanced mechanical properties. J. Mater. Chem. B 2020, 8, 7785-7791. [CrossRef]

16. Hou, S.-S.; Fan, N.-S.; Tseng, Y.-C.; Jan, J.-S. Self-Assembly and Hydrogelation of Coil-Sheet Poly (l-lysine)-block-poly (l-threonine) Block Copolypeptides. Macromolecules 2018, 51, 8054-8063. [CrossRef]

17. Gaspard, J.; Silas, J.A.; Shantz, D.F.; Jan, J.-S. Supramolecular assembly of lysine-b-glycine block copolypeptides at different solution conditions. Supramol. Chem. 2010, 22, 178-185. [CrossRef]

18. Huang, Y.-C.; Arham, M.; Jan, J.-S. Alkyl chain grafted poly (l-lysine): Self-assembly and biomedical application as carriers. Soft Matter 2011, 7, 3975-3983. [CrossRef]

19. Zhang, Y.-X.; Chen, Y.-F.; Shen, X.-Y.; Hu, J.-J.; Jan, J.-S. Reduction-and pH-Sensitive lipoic acid-modified Poly (l-lysine) and polypeptide/silica hybrid hydrogels/nanogels. Polymer 2016, 86, 32-41. [CrossRef]

20. Cheng, J.; Deming, T.J. Synthesis of polypeptides by ring-opening polymerization of $\alpha$-amino acid N-carboxyanhydrides. In Peptide-Based Materials; Deming, T., Ed.; Springer: Berlin/Heidelberg, Germany, 2011; Volume 310, p. 126.

21. Aoi, K.; Tsutsumiuchi, K.; Okada, M. Glycopeptide Synthesis by an. alpha.-Amino Acid N-Carboxyanhydride (NCA) Method: Ring-Opening Polymerization of a Sugar-Substituted NCA. Macromolecules 1994, 27, 875-877. [CrossRef]

22. Jiang, J.; Zhang, X.; Fan, Z.; Du, J. Ring-Opening Polymerization of N-Carboxyanhydride-Induced Self-Assembly for Fabricating Biodegradable Polymer Vesicles. ACS Macro Lett. 2019, 8, 1216-1221. [CrossRef]

23. Zhou, P.; Dai, X.-G.; Kong, J.; Ling, J. Synthesis of Well-defined Poly (tetrahydrofuran)-b-Poly (a-amino acid) s via Cationic Ring-opening Polymerization (ROP) of Tetrahydrofuran and Nucleophilic ROP of N-thiocarboxyanhydrides. Chin. J. Polym. Sci. 2021, 39, 702-708. [CrossRef]

24. Marzano, M.; Falanga, A.P.; Marasco, D.; Borbone, N.; D’Errico, S.; Piccialli, G.; Roviello, G.N.; Oliviero, G. Evaluation of an analogue of the marine $\varepsilon$-PLL peptide as a ligand of G-quadruplex DNA structures. Mar. Drugs 2020, 18, 49. [CrossRef] [PubMed]

25. Deming, T.J. Synthesis of side-chain modified polypeptides. Chem. Rev. 2016, 116, 786-808. [CrossRef] 
26. Hou, S.-S.; Hsu, Y.-Y.; Lin, J.-H.; Jan, J.-S. Alkyl-poly (L-threonine)/cyclodextrin supramolecular hydrogels with different molecular assemblies and gel properties. ACS Macro Lett. 2016, 5, 1201-1205. [CrossRef]

27. Hsiao, L.-W.; Lai, Y.-D.; Lai, J.-T.; Hsu, C.-C.; Wang, N.-Y.; Steven, S.-S.W.; Jan, J.-S. Cross-linked polypeptide-based gel particles by emulsion for efficient protein encapsulation. Polymer 2017, 115, 261-272. [CrossRef]

28. Shen, X.-Y.; Tang, C.-C.; Jan, J.-S. Synthesis and hydrogelation of star-shaped poly (L-lysine) polypeptides modified with different functional groups. Polymer 2018, 151, 108-116. [CrossRef]

29. Shen, Y.; Zhang, S.; Wan, Y.; Fu, W.; Li, Z. Hydrogels assembled from star-shaped polypeptides with a dendrimer as the core. Soft Matter 2015, 11, 2945-2951. [CrossRef] [PubMed]

30. Murphy, R.; Borase, T.; Payne, C.; O’Dwyer, J.; Cryan, S.-A.; Heise, A. Hydrogels from amphiphilic star block copolypeptides. RSC Adv. 2016, 6, 23370-23376. [CrossRef]

31. Tang, C.-C.; Zhang, S.-H.; My Phan, T.H.; Tseng, Y.-C.; Jan, J.-S. Block length and topology affect self-assembly and gelation of poly(L-lysine)-block-poly(S-benzyl-1-cysteine) block copolypeptides. Polymer 2021, 228, 123891. [CrossRef]

32. Chen, Y.-F.; Lai, Y.-D.; Chang, C.-H.; Tsai, Y.-C.; Tang, C.-C.; Jan, J.-S. Star-shaped polypeptides exhibit potent antibacterial activities. Nanoscale 2019, 11, 11696-11708. [CrossRef]

33. Breedveld, V.; Nowak, A.P.; Sato, J.; Deming, T.J.; Pine, D.J. Rheology of block copolypeptide solutions: Hydrogels with tunable properties. Macromolecules 2004, 37, 3943-3953. [CrossRef]

34. Zhang, D.; Qi, D.; Wang, J.; Yu, S.; He, C.; Deng, M. Effects of ethyl-L-glutamated and phenylalanine ratio/sequence on the secondary structure and gelation properties of their PEGylated copolymers. Polymer 2020, 191, 122276. [CrossRef]

35. Thornton, P.D.; Billah, S.M.R.; Cameron, N.R. Enzyme-Degradable Self-Assembled Hydrogels From Polyalanine-Modified Poly (ethylene glycol) Star Polymers. Macromol. Rapid Commun. 2013, 34, 257-262. [CrossRef] [PubMed]

36. Kim, M.S.; Dayananda, K.; Choi, E.K.; Park, H.J.; Kim, J.S.; Lee, D.S. Synthesis and characterization of poly (l-glutamic acid)block-poly (l-phenylalanine). Polymer 2009, 50, 2252-2257. [CrossRef]

37. Kousar, A.; Liu, J.; Mehwish, N.; Wang, F.; Dang-i, A.; Feng, C. pH-Regulated supramolecular chirality of phenylalanine-based hydrogels. Mater. Today Chem. 2019, 11, 217-224. [CrossRef]

38. Triftaridou, A.I.; Chécot, F.; Iliopoulos, I. Poly (N, N-dimethylacrylamide)-block-Poly (L-lysine) Hybrid Block Copolymers: Synthesis and Aqueous Solution Characterization. Macromol. Chem. Phys. 2010, 211, 768-777. [CrossRef]

39. Cheng, Y.; He, C.; Xiao, C.; Ding, J.; Zhuang, X.; Huang, Y.; Chen, X. Decisive role of hydrophobic side groups of polypeptides in thermosensitive gelation. Biomacromolecules 2012, 13, 2053-2059. [CrossRef]

40. Zhang, S.; Fu, W.; Li, Z. Supramolecular hydrogels assembled from nonionic poly (ethylene glycol)-b-polypeptide diblocks containing OEGylated poly-L-glutamate. Polym. Chem. 2014, 5, 3346-3351. [CrossRef]

41. Chen, B.-Y.; Huang, Y.-C.; Jan, J.-S. Molecular assembly of alkyl chain-grafted poly (L-lysine) tuned by backbone chain length and grafted alkyl chain. RSC Adv. 2015, 5, 22783-22791. [CrossRef]

42. Chen, B.-Y.; Huang, Y.-F.; Huang, Y.-C.; Wen, T.-C.; Jan, J.-S. Alkyl chain-grafted poly (L-lysine) vesicles with tunable molecular assembly and membrane permeability. ACS Macro Lett. 2014, 3, 220-223. [CrossRef]

43. Chen, Y.-F.; Shiau, A.-L.; Chang, S.-J.; Fan, N.-S.; Wang, C.-T.; Wu, C.-L.; Jan, J.-S. One-dimensional poly (L-lysine)-block-poly (L-threonine) assemblies exhibit potent anticancer activity by enhancing membranolysis. Acta Biomater. 2017, 55, $283-295$. [CrossRef]

44. Holowka, E.P.; Pochan, D.J.; Deming, T.J. Charged polypeptide vesicles with controllable diameter. J. Am. Chem. Soc. 2005, 127, 12423-12428. [CrossRef]

45. Nowak, A.P.; Breedveld, V.; Pakstis, L.; Ozbas, B.; Pine, D.J.; Pochan, D.; Deming, T.J. Rapidly recovering hydrogel scaffolds from self-assembling diblock copolypeptide amphiphiles. Nature 2002, 417, 424-428. [CrossRef] [PubMed]

46. Hoffman, R.L. Explanations for the cause of shear thickening in concentrated colloidal suspensions. J. Rheol. 1998, 42, 111-123. [CrossRef]

47. Chu, B. Laser Light Scattering: Basic Principles and Practice; Courier Corporation: North Chelmsford, MA, USA, 2007.

48. Tsai, Y.-L.; Tseng, Y.-C.; Chen, Y.-M.; Wen, T.-C.; Jan, J.-S. Zwitterionic polypeptides bearing carboxybetaine and sulfobetaine: Synthesis, self-assembly, and their interactions with proteins. Polym. Chem. 2018, 9, 1178-1189. [CrossRef]

49. Chan, B.A.; Xuan, S.; Horton, M.; Zhang, D. 1, 1, 3, 3-Tetramethylguanidine-promoted ring-opening polymerization of N-butyl N-carboxyanhydride using alcohol initiators. Macromolecules 2016, 49, 2002-2012. [CrossRef]

50. Jommanee, N.; Chanthad, C.; Manokruang, K. Preparation of injectable hydrogels from temperature and pH responsive grafted chitosan with tuned gelation temperature suitable for tumor acidic environment. Carbohydr. Polym. 2018, 198, 486-494. [CrossRef] [PubMed]

51. Kitagawa, M.; Maeda, T.; Hotta, A. PEG-based nanocomposite hydrogel: Thermo-responsive sol-gel transition and degradation behavior controlled by the LA/GA ratio of PLGA-PEG-PLGA. Polym. Degrad. Stab. 2018, 147, 222-228. [CrossRef] 